\title{
Diagnosis of Bezoar-induced small-bowel obstruction on sonography in emergency department
}

\author{
Zn-Jui Liu ${ }^{1}$, Chii-Hwa Chern ${ }^{2,3}$, Jen-Dar Chen ${ }^{3,4}$, Wen-Han Chang ${ }^{1,5,6,7,8}$, Pang-Yen Chen ${ }^{1,3}$ \\ ${ }^{1}$ Department of Emergency Medicine, Mackay Memorial Hospital, Taipei, Taiwan; ${ }^{2}$ Department of Emergency Medicine, Veterans General \\ Hospital-Taipei, Taipei, Taiwan; ${ }^{3}$ School of Medicine, National Yang-Ming University, Taipei, Taiwan; ${ }^{4}$ Department of Radiology, Far Eastern \\ Memorial Hospital, Taipei, Taiwan; ${ }^{5}$ Department of Medicine, Mackay Medical College, Taipei, Taiwan; ${ }^{6}$ Mackay Junior College of Medicine, \\ Nursing, and Management, Taipei, Taiwan; ${ }^{7}$ Institute of Mechatronic Engineering, National Taipei University of Technology, Taipei, Taiwan; \\ ${ }^{8}$ Institute of Injury Prevention and Control, Taipei Medical University, Taipei, Taiwan \\ Correspondence to: Pang-Yen Chen, MD. Department of Emergency Medicine, Mackay Memorial Hospital, Taipei, Taiwan. \\ Email: pongyen2000@gmail.com.
}

\begin{abstract}
Bezoars are defined as indigestible foreign materials impacting in the gastrointestinal tract, which are classified according to what they are composed of. Small intestinal obstruction caused by bezoar impaction was a rare disease. However, it has become more and more recognized since gastrectomy with truncal vagotomy was introduced into the treatment of peptic ulcer disease. Computerized tomography ("CT") is still the imaging modality of choice in preoperative diagnosis of small bowel obstruction caused by bezoars. Although ultrasound has less sensitivity and specificity in detecting bezoars as compared with CT, it still can reveal bezoars in $88 \%$ of patients. Some case reports have addressed the role of sonographic detection of small intestinal phytobezoar. Ultrasonography may be a more available and time saving tool when used as a "screening" modality at emergency department for patients with mild persistent nonspecific abdominal pain but without definite peritoneal signs, especially when presence of past history of gastrectomy with truncal vagotomy.
\end{abstract}

Keywords: Bezoar; small-bowel obstruction; sonography

Received: 08 September 2018; Accepted: 29 November 2018; Published: 27 December 2018.

doi: $10.21037 /$ ht.2018.12.01

View this article at: http://dx.doi.org/10.21037/ht.2018.12.01

\section{Introduction}

Bezoars are defined as indigestible foreign materials impacting in the gastrointestinal tract, which are classified according to what they are composed of. Small intestinal obstruction caused by bezoar impaction was a rare disease. However, it has become more and more recognized since gastrectomy with truncal vagotomy was introduced into the treatment of peptic ulcer disease. This is a typical case with a medical history of radical subtotal gastrectomy with Billroth II reconstruction and truncal vagotomy for gastric adenocarcinoma about one year ago, and symptoms of intestine obstruction occurred. It reminds us that sonography is still a practical tool in screening small intestine obstruction due to bezoar impaction.

\section{Case presentation}

An 83-year-old man presented to our emergency department (ED) due to right abdominal intermittent cramping pain for several days. He had a past history of radical subtotal gastrectomy with Billroth II reconstruction and truncal vagotomy for gastric adenocarcinoma about one year ago. There was anorexia and nausea sensation after meal. The amount of stool passage decreased gradually. Physical examination revealed mild tenderness over the right upper and middle abdomen, but without rebounding pain or muscle guarding. The bowel sound was hyperperistalsis. Laboratory data only showed mild leukocytosis (white blood cell count: 11,200/cumm, segmented neutrophil: $71.7 \%$, lymphocyte: $23.2 \%$, 


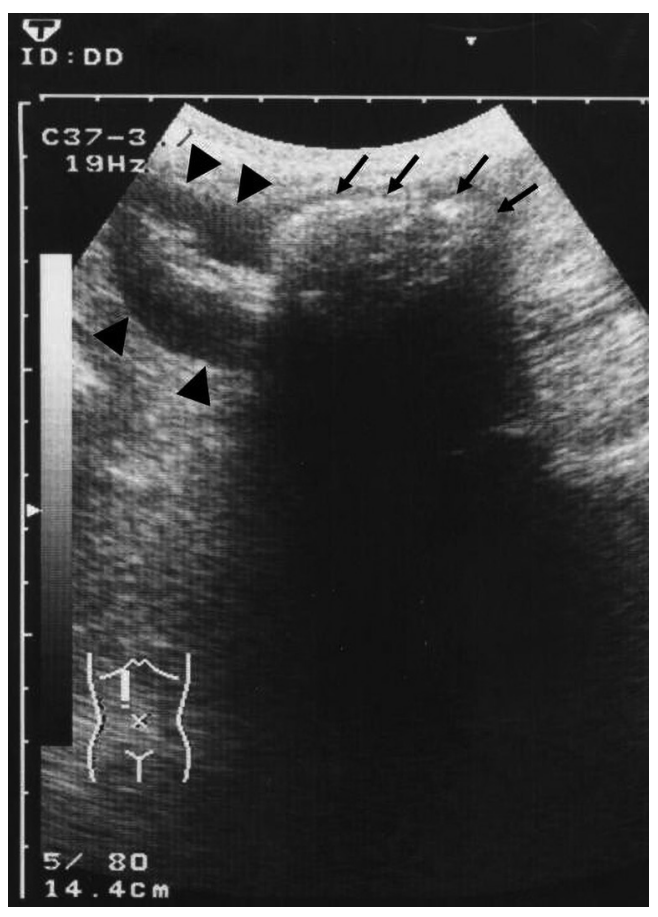

Figure 1 The ultrasound image of right upper abdomen in sagittal plane showed an intraluminal hyperechoic arclike mass with posterior acoustic shadow within a segment of dilated bowel loop (arrows), associated with adjacent bowel wall thickening just proximal to the obstruction level (arrow heads).

monocyte: $3.8 \%$ ) and slightly elevated C-reactive protein $(5.6 \mathrm{mg} / \mathrm{dL})$. Sonography of abdomen was performed with Toshiba SSA-340A ECOOCEE Ultrasound System and a 3.75-MHz Convex transducer (Toshiba PVE-375M). An intraluminal hyperechoic arclike mass with posterior acoustic shadow within a segment of dilated bowel loop was revealed. Adjacent bowel wall thickening just proximal to the obstruction level was also noted (Figure 1). An abdominal computerized tomography ("CT") for the patient demonstrated a well-defined oval intraluminal mottled mass of mixed gas and soft tissue densities impacted within a dilated loop of the ileum with bowel wall thickening proximal to the level of bezoar. It caused collapse of the distal small bowel, and dilatation of small bowel proximal to the bezoar (Figure 2). Partial intestine obstruction due to bezoar impaction was diagnosed. After discussion with the surgeon, an operation was seemed to be unavoidable, if condition went worse. Yet, conservative treatment with close observation was still tried first by gradual hydration via intravenous injection and restriction of food intake. The patient kept nothing by mouth [Nil Per Os (NPO)],

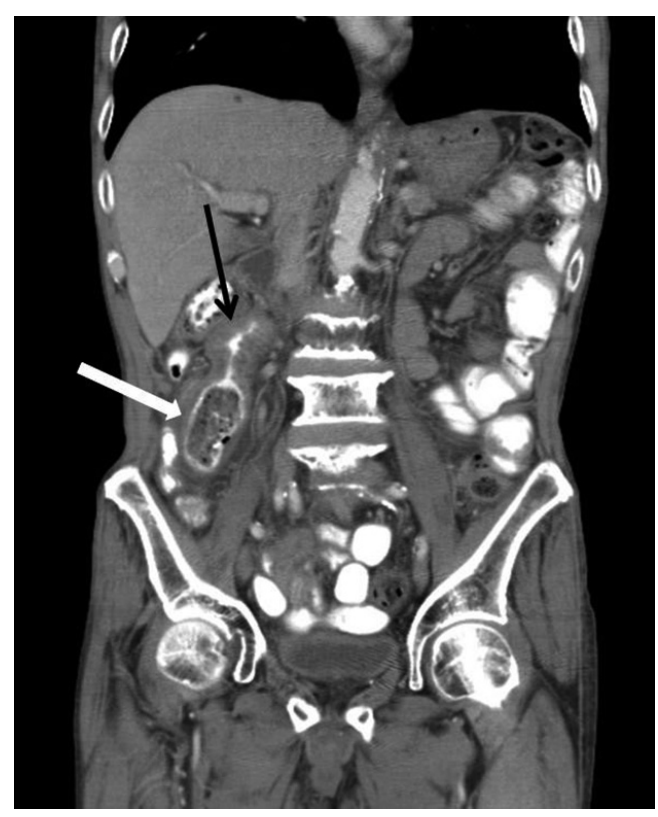

Figure 2 Coronary reformatted image of abdominal CT scan demonstrated a well-defined oval intraluminal mottled mass of mixed gas and soft tissue densities impacted within a dilated loop of the ileum (white arrow). Bowel wall thickening was also demonstrated proximal to the level of bezoar (black arrow).

and peripheral parenteral nutrition (PPN) supplement was administered. He recovered gradually and low residual diet was tried. He tolerated well. There was no more abdominal discomfort or obstruction signs. Thus, the plan of operation was cancelled. Finally, he was discharged one week after admission due to stable condition.

\section{Discussion}

There are many different causes of small bowel obstruction. Extrinsic compression due to post-operative adhesions or hernias is more common. Post-operative adhesions now cause almost three-fourths of small bowel obstruction, although hernias were once the most common cause of it. It's due to that the survival rate and numbers of patients of laparotomy increased a lot in recent decades. Less frequently, intrinsic lesions of normal bowel lumen may be the cause of small bowel obstruction as well (1).

Bezoar impaction is a rare cause of obstruction of normal bowel lumen. Bezoars are defined as indigestible foreign materials, impacting in the gastrointestinal tract, which are classified according to what they are composed of. Trichobezoars and phytobezoars are the most frequent 
forms (2). They are respectively composed of hairs or vegetable and fruit fibers. History taking is very important in the diagnosis of bezoar-induced intestinal obstruction. Eighty percent of trichobezoars occur in people under age of 30 years, and more than $90 \%$ of them are females (3). Trichobezoars are associated with mental retardation, pica, and trichotillomania. On the other hand, about $75 \%$ of patients with phytobezoar have a past history of subtotal gastrectomy or gastrojejunostomy (4). A history of excessive intake of vegetable fibers was revealed in about $40 \%$ cases of phytobezoar (5). The common symptoms of bezoarinduced intestinal obstruction are early satiety, body weight loss, abdominal pain, nausea and vomiting (6). It could be mild or severe. Sometimes, a palpable mass may be noted. $\mathrm{CT}$ is still the imaging modality of choice in preoperative diagnosis of small bowel obstruction caused by bezoars. Although ultrasound has less sensitivity and specificity in detecting bezoars as compared with CT. It still can reveal bezoars in $88 \%$ of patients (4). In addition, ultrasound may be a more available and time saving tool when used as a "screening" modality at ED for patients with mild persistent nonspecific abdominal pain but without definite peritoneal signs, especially when presence of past history of gastrectomy with truncal vagotomy. Schmutz et al. reported that sonography had a specificity of $82.1 \%$ and sensitivity of $95 \%$ in detecting small bowel obstruction. It appears accurate in confirming a small bowel obstruction and in determining the etiology of small bowel obstruction. During the sonography, hyperperistaltic fluid-filled loops with diameter greater than $25 \mathrm{~mm}$ in the jejunum, greater than $15 \mathrm{~mm}$ in the ileum, and collapsed colonic lumen suggest of small bowel obstruction. In contradistinction, distended small bowel loops with abundant gas, hypoperistaltic motion and a colonic lumen filled with gas, fluid or stool suggest of paralytic ileus $(7,8)$.

Intussusceptions and primary small bowel carcinomas may be characterized as "target" or "bull's eye" lesion in sonographic patterns (8). Pneumobilia and direct visualisation of ectopic stone are the more specific finding of gallstone ileus (9). Ultrasound images of bezoar impacted in small bowel may show a long intraluminal, hyperechogenic mass with intense posterior acoustic shadowing. The lumen in front of the impacted bezoar may be dilated with accumulated fluid. Sometimes, a segment of proximal bowel wall swelling may be found. The sonographic appearance of this case presented the same pictures.

Some case reports have addressed the role of sonographic detection of small intestinal phytobezoar (10-12).
Sonography may be appropriate for pregnant patients or as a bedside real-time tool for the critically ill patients at ED, although it's not as helpful as CT for the diagnosis of location, cause, and possible strangulation of small intestinal obstruction. Evaluation of intestines should be included in the ultrasound training program of emergency medicine curriculum.

\section{Acknowledgments}

Funding: None.

\section{Footnote}

Conflicts of Interest: WHC serves as an Editor-in-Chief of Health Technology. The other authors have no conflicts of interest to declare.

Ethical Statement: The authors are accountable for all aspects of the work in ensuring that questions related to the accuracy or integrity of any part of the work are appropriately investigated and resolved. All procedures performed in studies involving human participants were in accordance with the Declaration of Helsinki (as revised in 2013). Written informed consent was obtained from the patient for publication of this manuscript and any

Open Access Statement: This is an Open Access article distributed in accordance with the Creative Commons Attribution-NonCommercial-NoDerivs 4.0 International License (CC BY-NC-ND 4.0), which permits the noncommercial replication and distribution of the article with the strict proviso that no changes or edits are made and the original work is properly cited (including links to both the formal publication through the relevant DOI and the license). See: https://creativecommons.org/licenses/by-nc-nd/4.0/.

\section{References}

1. Deutsch AA, Eviatar E, Gutman H, et al. Small bowel obstruction: a review of 264 cases and suggestions for management. Postgrad Med J 1989;65:463-7.

2. Ogawa K, Kamimura K, Mizuno K, et al. The Combination Therapy of Dissolution Using Carbonated Liquid and Endoscopic Procedure for Bezoars: Pragmatical and Clinical Review. Gastroenterol Res Pract 2016;2016:7456242.

3. DeBakey M, Ochsner A. Bezoars and concretions. Surgery 
1938;4:934-63.

4. Ripollés T, García-Aguayo J, Martínez MJ, et al. Gastrointestinal bezoars: sonographic and CT characteristics. AJR Am J Roentgenol 2001;177:65-9.

5. Escamilla C, Robles-Campos R, Parrilla-Paricio P, et al. Intestinal obstruction and bezoars. J Am Coll Surg 1994;179:285-8.

6. Kamimura K, Evans DC, Mizuno KI, et al. Diagnosis and Management of Gastrointestinal Foreign Bodies. Gastroenterol Res Pract 2016;2016:5692650.

7. Schmutz GR, Benko A, Fournier L, et al. Small bowel obstruction: role and contribution of sonography. Eur Radiol 1997;7:1054-8.

8. Morgan CL, Trought WS, Oddson TA, et al. Ultrasound doi: 10.21037/ht.2018.12.01

Cite this article as: Liu ZJ, Chern $\mathrm{CH}$, Chen JD, Chang WH, Chen PY. Diagnosis of Bezoar-induced small-bowel obstruction on sonography in emergency department. Health Technol 2018;2:5. patterns of disorders affecting the gastrointestinal tract. Radiology 1980;135:129-35.

9. Lassandro F, Gagliardi N, Scuderi M, et al. Gallstone ileus analysis of radiological findings in 27 patients. Eur J Radiol 2004;50:23-9.

10. Ko YT, Lim JH, Lee DH, Yoon Y. Small intestinal phytobezoars: sonographic detection. Abdom Imaging 1993;18:271-3.

11. Yildirim B, Gürkaynak G, Akyol D, et al. Ultrasonographic diagnosis of small intestinal phytobezoar. J Clin Ultrasound 1996;24:213-6.

12. Oktar SO, Erbaş G, Yücel C, et al. Closed perforation of the small bowel secondary to a phytobezoar: imaging findings. Diagn Interv Radiol 2007;13:19-22. 